\section{El consentimiento informado en la investigación pediátrica}

\section{Informed Consent in pediatric research}

Flores-Pérez $\mathrm{J}^{1}$, Monroy-Santoyo $\mathrm{S}^{2}$, Ruíz-García $\mathrm{M}^{3}$, González-Zamora JF${ }^{4}$, Niembro-Zúñiga $\mathrm{AM}^{5}$, Greenawalt-Rodríguez $\mathrm{SR}^{6}$, Sánchez-Guerrero $\mathrm{O}^{7}$, Priego y Romo $\mathrm{J}^{8}$, De Rubens-Figueroa $\mathrm{J}^{9}$, Vázquez-Cárdenas $\mathrm{C}^{10}$, EscamillaVilla $A^{11}$, Palacios-López CG $^{12}$

\section{INTRODUCCIÓN}

Los informes de abusos cometidos durante la Segunda Guerra Mundial, en sujetos sometidos a experimentos biomédicos, son el punto de referencia para la formulación de las reglas básicas a fin de llevar a cabo investigación en seres humanos. Así, surge el Informe Belmont, en el que quedan plasmados los tres principios éticos básicos: respeto por la persona, cuya expresión máxima es la autonomía, el beneficio y la justicia.

La aplicación de dichos principios ligados a la investigación en seres humanos lleva a considerar al consentimiento informado como la parte medular para la preservación de la autonomía de los participantes. Más que un documento, el consentimiento informado es un proceso dinámico entre ambas partes: investigador y sujeto de investigación, donde se deben conjuntar la información suficiente, veraz, clara y oportuna, con la comprensión y la voluntariedad del sujeto.

En México, tanto el Reglamento de La Ley General de Salud en Materia de Investigación para la Salud, en su Artículo 14, fracción V, como la Norma Oficial Mexicana NOM-012-SSA3-2012, establecen la obligatoriedad de otorgar consentimiento informado y por escrito del sujeto de investigación.

\section{DEFINICIÓN}

El Consentimiento Informado es un documento de carácter legal, por medio del cual el investigador y el sujeto de la in-

\footnotetext{
${ }^{1}$ Laboratorio de Farmacología.

${ }^{2}$ Laboratorio de Cirugía Experimental. ${ }^{3}$ Neurología.

${ }^{4}$ Laboratorio de Cirugía Experimental.

${ }^{5}$ Oncología.

${ }^{6}$ Consulta externa Pediatría.

${ }^{7}$ Departamento de Psiquiatría.

${ }^{8}$ Comunicación Social.

${ }^{9}$ Servicio de Cardiología.

${ }^{10}$ Vocal de la Comunidad.

${ }^{11}$ Departamento de lo Consecutivo.

${ }^{12}$ Servicio de Dermatología.
}

Comité de Ética en Investigación del Instituto Nacional de Pediatría, Ciudad de México.

Recibido: 13 de septiembre del 2016

Aceptado: 1 de noviembre del 2016

Correspondencia

Palacios-López Carolina Guadalupe

caroderma@yahoo.com.mx

Este artículo debe citarse como

Flores-Pérez J, Monroy-Santoyo S, Ruíz-García M, González-Zamora JF, Niembro-Zúñiga AM, Greenawalt-Rodríguez SR et al. El consentimiento informado en la investigación pediátrica. Acta Pediatr Mex. 2017;38(2):125-127.

DOI: http://dx.doi.org/10.18233/APM38No2pp125-1271364 
vestigación convienen y aceptan las reglas y condiciones básicas a las que se sujetan ambas partes, en el proceso y desarrollo del proyecto de investigación.

\section{OBJETIVO}

Favorecer la participación de los sujetos de investigación en edad pediátrica, mediante la comprensión de los procedimientos a realizar, beneficios, alcances, alternativas y riesgos asociados al proyecto, con la finalidad de que sus padres o tutores legales lleguen a una decisión voluntaria de aceptación, rechazo o eventual retiro del niño del estudio.

\section{¿QUÉ DEBE CONTENER EL CONSENTI- MIENTO INFORMADO?}

1. Invitación al proyecto con lenguaje sencillo y claro.

2. Propósito u objetivos de la investigación.

3. Tipo de intervención a realizarse en el proyecto.

4. Por qué el niño es o no sujeto de esta investigación.

5. Establecer con claridad la voluntariedad de su participación.

6. Procedimientos y protocolo que se llevarán a cabo.

7. Posibles riesgos asociados a la maniobra de investigación.

8. Beneficios individuales o colectivos que pueden obtenerse.

9. Pautas de confidencialidad y divulgación de resultados.

10. Derecho a no participar o a retirarse en cualquier momento de la investigación.
11. La negativa a participar, no implica de manera alguna la alteración de la atención médica que regularmente se brinda al paciente.

12. Información del investigador para su localización en caso de requerir contactarlo.

\section{¿CÓMO OBTENER EL CONSENTIMIENTO INFORMADO?}

Se debe considerar siempre que el Consentimiento Informado es un documento legal, por lo que todos los estudios de investigación demandan este manuscrito. En el caso de la investigación en pediatría se requiere la autorización por escrito de ambos padres o tutor legal del niño involucrado en el estudio. De manera excepcional, y de acuerdo a cada caso, se permite la autorización sólo de uno de los padres. Para niños mayores de 12 años se sugiere la realización concomitante de la Carta de Asentimiento Informado.

El personal de salud involucrado en el desarrollo del proyecto de investigación es el encargado de obtener el Consentimiento Informado. Debe estar capacitado y en absoluta disponibilidad para brindar toda la información que los padres requieran, a fin de aceptar o rechazar de manera voluntaria su participación en el estudio.

Se deben explicar con claridad los alcances de ese consentimiento informado en particular, ya que el paciente tiene derecho a negar su autorización a algunos apartados sin que influya en su participación dentro de la investigación; por ejemplo, el resguardo de sus datos personales, muestras de ADN o de la negativa a conocer sus resultados.

Una vez firmado el documento se deberá entregar una copia a los padres y siempre tener en mente que el consentimiento informado no es un contrato, y tanto el investigador como los 
padres tienen derecho a retirarse en cualquier momento sin dar explicaciones.

\section{CONSIDERACIONES FINALES}

La formulación y obtención del consentimiento informado en investigación pediátrica de ninguna manera supone una limitante para la realización de proyectos de investigación, los cuales son indispensables para el desarrollo de las sociedades. La trascendencia del consentimiento informado va más allá de ser un mero documento legal; sus implicaciones van en ambas direcciones, al proteger la autonomía del niño y la investigación biomédica en curso.

Se puede obtener una guía para la realización del formato de consentimiento informado en: http://www.pediatria.gob.mx/investigacion/archivos/consentimiento_informado.pdf

\section{LECTURAS RECOMENDADAS}

1. Consejo de Organizaciones Internacionales de las Ciencias Médicas (CIOMS) en colaboración con la Organización Mundial de la Salud (OMS). Pautas Clínicas internacionales para la investigación biomédica en seres humanos. Chile Mayo 2003: 37-45 ISBN 956-7938-03-2.

2. Comisionado de autorización sanitaria de la Comisión Federal para la protección contra riesgos sanitarios Dr. Federico Alberto Argüelles Tello Lineamientos para cumplir las Buenas Prácticas Clínicas en la Investigación para la Salud. COFEPRIS 5 de mayo de 2012.

3. Norma Oficial Mexicana NOM-012-SSA3-2012 establece los Criterios para la ejecución de proyectos de investigación para la Salud en Seres Humanos.

4. Zawitowski CA, Frader JE. Ethical problems in pediatric critical care consent. CriticalCareMed. 2003:31(Supl);407410.

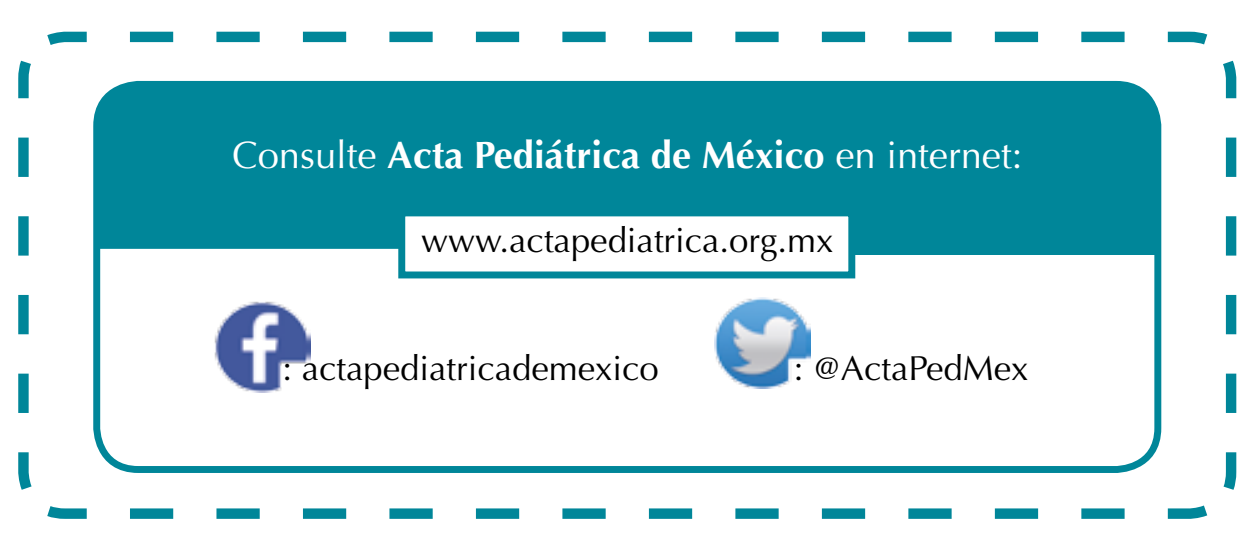

